\title{
Society position statement
}

\section{Canadian Cardiovascular Society/Canadian Anesthesiologists' Society/Canadian Heart Rhythm Society joint position statement on the perioperative management of patients with implanted pacemakers, defibrillators, and neurostimulating devices}

\author{
Jeff S. Healey, MD • Richard Merchant, MD • Chris Simpson, MD • \\ Timothy Tang, MD • Marianne Beardsall, MN/NP • Stanley Tung, MD • \\ Jennifer A. Fraser, RN • Laurene Long, RN • Janet M. van Vlymen, MD • \\ Pirjo Manninen, MD • Fiona Ralley, MBBCh • Lashmi Venkatraghavan, MD • \\ Raymond Yee, MD • Bruce Prasloski, MD • Shubhayan Sanatani, MD • \\ François Philippon, MD
}

Published online: 10 March 2012

(C) Canadian Anesthesiologists' Society and Canadian Cardiovascular Society 2012

\begin{abstract}
Purpose There are more than 200,000 Canadians living with permanent pacemakers or implantable defibrillators, many of whom will require surgery or invasive procedures each year. They face potential hazards when undergoing surgery; however, with appropriate planning and education of operating room personnel, adverse device-related outcomes should be rare. This joint position statement from
\end{abstract}

This article is published concurrently in the Canadian Journal of Anesthesia and the Canadian Journal of Cardiology with the express agreement of all authors as well as the editors of both journals.

This statement was developed following a thorough consideration of medical literature and the best available evidence and clinical experience. It represents the consensus of a Canadian panel comprised of multidisciplinary experts on this topic with a mandate to formulate disease-specific recommendations. These recommendations are aimed to provide a reasonable and practical approach to care for specialists and allied health professionals obliged with the duty of bestowing optimal care to patients and families, and can be subject to change as scientific knowledge and technology advance and as practice patterns evolve. The statement is not intended to be a substitute for physicians using their individual judgment in managing clinical care in consultation with the patient, with appropriate regard to all the individual circumstances of the patient, diagnostic and treatment options available and available resources. Adherence to these recommendations will not necessarily produce successful outcomes in every case.

\section{J. S. Healey, MD (ه)}

Population Health Research Institute, McMaster University,

Room C3-121, David Braley CVSRI, 237 Barton St East,

Hamilton, ON L8L 2X2, Canada

e-mail: Jeff.Healey@phri.ca the Canadian Cardiovascular Society (CCS) and the Canadian Anesthesiologists' Society (CAS) has been developed as an accessible reference for physicians and surgeons, providing an overview of the key issues for the preoperative, intraoperative, and postoperative care of these patients.

Principal findings The document summarizes the limited published literature in this field, but for most issues, relies heavily on the experience of the cardiologists and anesthesiologists who contributed to this work. This position statement outlines how to obtain information about an individual's type of pacemaker or implantable defibrillator and its programming. It also stresses the importance of determining if a patient is highly pacemaker-dependent and proposes a simple approach for nonelective evaluation of dependency. Although the document provides a comprehensive list of the intraoperative issues facing these patients, there is a focus on electromagnetic interference resulting from electrocautery and practical guidance is given regarding the characteristics of surgery, electrocautery, pacemakers, and defibrillators which are most likely to lead to interference.

Conclusions The document stresses the importance of preoperative consultation and planning to minimize complications. It reviews the relative merits of intraoperative magnet use vs reprogramming of devices and gives examples of situations where one or the other approach is preferable.

R. Merchant, MD · S. Tung, MD · B. Prasloski, MD .

S. Sanatani, MD

University of British Columbia, Vancouver, BC, Canada 


\section{Résumé}

Objectif Plus de 200000 Canadiens vivent avec des stimulateurs cardiaques permanents ou des défibrillateurs implantables et plusieurs d'entre eux auront besoin d'une chirurgie ou de procédures effractives chaque année. Ils font face à des risques potentiels lorsqu'ils subissent une chirurgie. Cependant, par une planification et une formation appropriée du personnel de salle d'opération, les conséquences négatives liées aux dispositifs devraient être rares. Cet énoncé de position commune de la Société canadienne de cardiologie (SCC) et de la Société canadienne des anesthésiologistes (SCA) a été élaboré en tant que référence accessible aux médecins et aux chirurgiens, fournissant un aperçu des questions clés pour les soins préopératoires, peropératoires et postopératoires de ces patients.

Constatations principales Le document résume la littérature limitée de ce domaine, mais pour la plupart des questions, il repose largement sur l'expérience des cardiologues et des anesthésiologistes qui ont contribué à ce travail. Cet énoncé de position décrit comment obtenir des renseignements sur un stimulateur cardiaque adapté aux besoins individuels ou un défibrillateur implantable et sa programmation. Il souligne aussi l'importance de déterminer si un patient est grandement dépendant de son

C. Simpson, MD · J. M. van Vlymen, MD

Queen's University, Kingston, ON, Canada

T. Tang, MD

University of Calgary, Calgary, AB, Canada

M. Beardsall, MN/NP

Southlake Regional Health Centre, Newmarket, ON, Canada

M. Beardsall, MN/NP

University of Toronto, Toronto, ON, Canada

J. A. Fraser, RN

Peterborough Regional Cardiac Device Clinic, Peterborough,

ON, Canada

L. Long, RN

Hamilton Health Sciences, Hamilton, ON, Canada

P. Manninen, MD · L. Venkatraghavan, MD

University Health Network, University of Toronto, Toronto, ON, Canada

F. Ralley, MBBCh $\cdot$ R. Yee, MD

University of Western Ontario, London, ON, Canada

F. Philippon, MD

Université Laval, Quebec City, QC, Canada stimulateur cardiaque et propose une approche simple pour l'évaluation non élective de dépendance. Même si le document fournit une liste exhaustive des questions peropératoires auxquelles font face ces patients, l'accent est mis sur l'interférence électromagnétique résultant de l'electrocautère, et des conseils pratiques sont donnés en ce qui concerne les caractéristiques de la chirurgie, de l'électrocautère, des stimulateurs cardiaques et des défibrillateurs, lesquels sont les plus susceptibles de mener à une interférence.

Conclusions Le document souligne l'importance d'une consultation préopératoire et d'une planification pour minimiser les complications. Il revoit la pertinence relative à l'utilisation d'un aimant peropératoire par rapport à la reprogrammation des dispositifs et donne des exemples de situations où l'une ou l'autre des approches est préférable.

Since the introduction of the first pacemaker more than 50 years ago, there has been a steady increase in the number of patients treated with increasingly complex implanted electronic devices (IEDs), including pacemakers, implantable cardioverter-defibrillators (ICDs), cardiac resynchronization devices, and a variety of neurostimulating devices. In North America, there are nearly 3 million individuals living with these cardiac rhythm devices (CRDs), and more than 250,000 new devices are implanted each year owing to an aging population and expanding indications for CRDs. ${ }^{1-3}$ Deep brain stimulators and other neurostimulators have become commonly accepted therapy for medication-refractory movement and neuropsychiatric disorders with more than 80,000 devices implanted worldwide..$^{4-6}$ It is thus commonplace for patients undergoing surgery to have IEDs.

Implanted devices and the operating room environment have both become more sophisticated, increasing the likelihood of interactions and making the perioperative management of these devices more complex. The goals of this position paper are to provide an overview of the perioperative issues related to the management of CRDs and neurostimulators and to discuss strategies for minimizing complications in the Canadian context.

The joint committee of the Canadian Anesthesiologists' Society (CAS) and the Canadian Heart Rhythm Society (CHRS) consists of members nominated by the CAS Standards Committee and the Canadian Cardiovascular Society (CCS)/CHRS. The joint committee includes anesthesiologists and cardiologists in private and academic practices from various geographic regions and paramedical health professionals involved in implantable electrical device care. The group's recommendations were reached 
by consensus of all members through an extensive review of previously published guidelines and current published evidence.

- What other guideline statements are available on this topic? The American Society of Anesthesiologists (ASA) has published ${ }^{7}$ and updated ${ }^{8}$ a practice advisory; as has the Heart Rhythm Society (HRS) ${ }^{9}$ and the Medicines and Healthcare products Regulatory Agency of the UK Department of Health. ${ }^{10}$

- Why was this guideline developed? How and why does this statement differ from existing guidelines? The current guidelines do not adequately address the Canadian context as it relates to the breadth of perioperative issues confronted by Canadian physicians as well as Canadian concerns in general, hence the direction to develop a practical clinically relevant document with broad reach appropriate for the Canadian context and with specific recommendations for management.

\section{Scope of document}

The focus of this document is on the management of patients with a CRD or other IED at the time of surgery or at the time of another invasive procedure (e.g., endoscopy, image-guided invasive treatment). Important device-related interactions may also occur at the time of magnetic resonance imaging, radiation therapy, or computerized tomography; however, these issues will not be specifically addressed in this document. This position statement is intended to convey the most important aspects of perioperative care in the Canadian context, to provide practical advice, and to expand on the existing information in the current guidelines to noncardiac devices. Because the better part of the literature deals with cardiac devices, we continue to focus primarily on these devices. However, we also review the issue of neurostimulating devices separately, recognizing that many concerns apply equally to this topic.

\section{Methodology and data sources}

This document was created by a joint committee, consisting of members of the CCS and the CAS. Members of this committee were chosen to include both physicians and nurses; individuals from tertiary care and community hospitals, those involved in adult and pediatric care, and including individuals directly involved in the management of implanted cardiac and neurostimulating devices. The relevant medical literature was reviewed independently by two committee members. Reference lists from review articles and position statements were also reviewed to identify important publications. All potentially relevant articles were circulated amongst the entire committee, whose entire membership was also free to propose additional relevant publications. A draft of the position statement was made by one of the committee co-chairs, with several sections written by committee members with relevant expertise. A series of teleconferences were conducted, at which time all committee members were invited to make comments regarding the current draft and revisions arising from the call were made to the document by the other co-chair. The final draft was circulated to all committee members for comment and approval.

We acknowledge that there are few high-quality publications in the medical literature to guide the perioperative management of CRDs. ${ }^{11}$ Most publications involve case reports, case series, consensus-based guidelines, and industry position statements. ${ }^{2,7,8,11,12}$ Because we did not find a single randomized trial comparing different management strategies, the recommendations in this document are based on a Grade C level of evidence.

\section{Perspective of the document}

The focus of this position statement is on patient-centred management when commenting on the most appropriate strategies to reduce perioperative risk. We appreciate that different perspectives may exist between staff in the operating room and staff in CRD clinics, and although the perspectives of both groups are important, the patient perspective supersedes. In addition to discussion of intraoperative issues, the document will address the preoperative and postoperative care of the patient.

\section{Potential perioperative issues with CRDs}

The most widely appreciated potential complication with CRDs in the operative setting is the inappropriate sensing of electromagnetic interference (EMI) caused by electrocautery. The consequence of such inappropriate sensing depends on the type of CRD involved, patient characteristics, and device settings. In general, however, the result may include:

1. No effect;

2. Inappropriate inhibition of pacing;

3. Inappropriate rapid pacing; or

4. Inappropriate sensing and triggering of ICD therapy (shock or rapid pacing, antitachycardia pacing [ATP]). 
There are some implications to device function associated with anesthesia and surgery.

1. Damage to or reprogramming of pacemaker systems. In a few case reports, these complications have been documented secondary to cautery use or associated with radiotherapy or radiofrequency ablation procedures, although the risk of these complications is considered quite low due to the incorporation of protective circuitry in modern pacemaker technology. 13 "Power on reset" mode-a simple backup pacing mode (typically VVI or VOO)—may be activated by disruption of a pacemaker's volatile electronic memory.

2. Anatomical consequences associated with surgical procedures-pneumothorax with thoracotomy or cardiac surgery: such results have been linked to changes in pacemaker function due to lead dislodgement, an increase in impedance, particularly with unipolar pacing systems, or an increase in the defibrillation threshold. $^{14,15}$

3. Physical damage to CRDs or leads through direct trauma; device or lead infection associated with perioperative bacteremia;

4. Physical damage (burn) to tissue associated with EMI conduction through leads; and

5. Complications associated with the failure to return devices to preoperative settings. ${ }^{16}$

6. It should be noted that vagotonic manoeuvres or agents (rapidly acting narcotics) may enhance conduction blockade and this effect is likely more pronounced in patients with sinus or atrioventricular node dysfunction. $^{17}$

7. Depending on the nature of the surgery, alterations in fluid balance, acid base status, electrolyte disturbances, and altered medication schedules can have an impact on device function and propensity to arrhythmias.

Given the limitations of available publications, it is difficult to estimate the true incidence of such complications. However, given the wide range of CRDs, patient characteristics, and institutional policies addressing perioperative management, risk and management must be considered on a case-by-case basis.

\section{Preoperative care}

Like all aspects of surgery, ensuring optimal CRD-related outcomes requires careful planning that should start as soon as possible after the decision is made to undertake surgery. The details of this planning will depend on the urgency of surgery (emergent vs elective), if surgery will be performed in an environment with onsite CRD support,
Table 1 Preoperative management

- Identify patient has CRD

- Identify responsible CRD clinic or physician

- Determine patient "dependency"

- Estimate likelihood of EMI depending on (1) nature of CRD and (2) surgery

- Estimate likelihood of CRD complications

- Develop collaborative plan to minimize risk

- Consider referral to higher-acuity institution in selected cases

$\mathrm{CRD}=$ cardiac rhythm device; $\mathrm{EMI}=$ electromagnetic interference

and if surgery will be conducted on an inpatient or outpatient basis. However, the following steps are critical in all cases (Table 1 ):

1. Ascertain that the patient has a CRD and identify the type of device (pacemaker, ICD, cardiac resynchronization device) and the device programming parameters. The specific device can be identified by consulting the patient's CRD clinic and/or physician, by reviewing the patient's medical record or device identification card, or in emergency situations, by examining characteristics of the implanted device on a chest radiograph (Fig. 1).

2. Identify the CRD clinic or physician responsible for management of the patient. In Canada, the patient's CRD clinic and/or physician is often not located at the same institution where surgery is planned. To the extent feasible, the CRD clinic or hospital should provide consulting physicians and anesthesiologists with access to patient and device information (Table 2). While gathering this information may present a challenge for emergency surgery in nonclinic hours, this should always be the goal. Most hospitals have medical records personnel available after hours, who can usually access relevant information. Communication with the patient's CRD clinic and/or physician can assist in determining if a device check is warranted or required prior to surgery based on recent follow-up data. In emergency cases where it is impossible to access the implanting referral centre, all CRD manufacturers provide 24-hr technical support services (see "Industry resources" section).

3. Determine the degree that the patient's cardiac rhythm is dependent on pacemaker function. This is essential to determine the likely consequences of inappropriate inhibition of pacing, and it is best accomplished by consulting the patient's CRD clinic or physician. A 12-lead electrocardiogram (ECG) should be performed prior to surgery to facilitate identifying patients who are paced but are not pacemaker-dependent (Fig. 2). In general, if the ECG demonstrates an intrinsically 

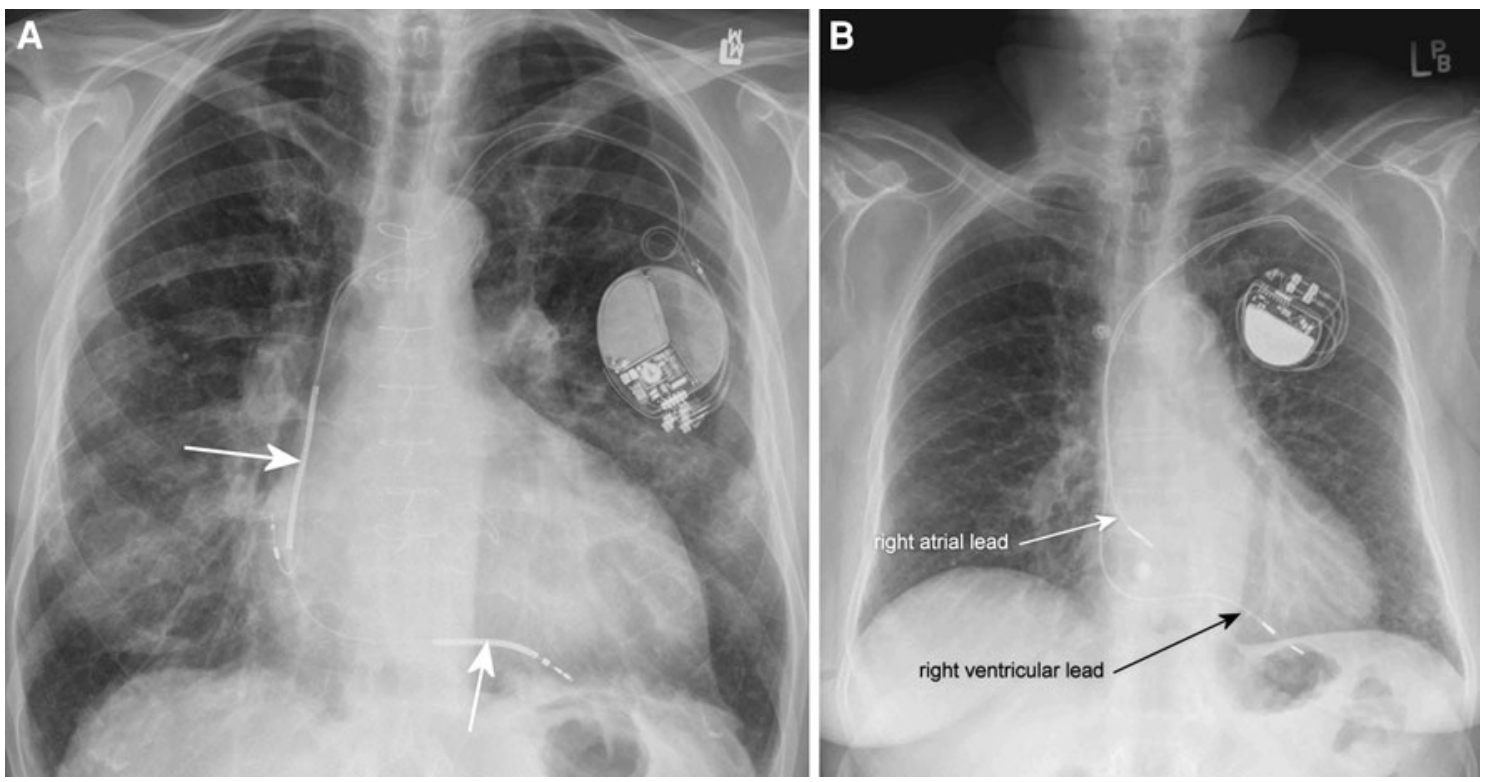

Fig. 1 Radiographic appearance of an implantable cardioverterdefibrillator (ICD) (A) and a cardiac pacemaker (B). The only reliable way to differentiate an ICD from a pacemaker is the presence

Table 2 Recommended minimum CRD data collection for perioperative assessment

- Device type, manufacturer, model

- Is the device or lead under recall or advisory?

- Date and hospital of implant and date of most recent follow-up

- Follow-up clinic and physician

- Minimum anticipated battery longevity

- Pacing dependency, pacing mode, and rate-modulation sensor

- Recent activity: atrial and ventricular pacing activity, VT, and VF detection

- Response to magnet (e.g., asynchronous pacing, suspended tachycardia detection)

- Expected response to magnet removal (e.g., resume original settings, other)

$\mathrm{CRD}=$ cardiac rhythm device; $\mathrm{VF}=$ ventricular fibrillation; $\mathrm{VT}=$ ventricular tachycardia

conducted rhythm (i.e., QRS complexes not preceded by a pacing artifact), then the patient is unlikely to be highly pacemaker-dependent. Clinicians should be careful not to overlook small bipolar pacing artifacts, particularly when QRS complexes are of left bundle branch-like morphology with negative complexes in leads II, III, and aVF (i.e., typical right ventricular apex pacing) (Fig. 2, A). In an emergency situation where a patient appears to be paced all the time, it is safest to assume that the patient is highly pacemakerdependent (Fig. 2, B). of shocking coil(s) (indicated by arrows) in the right ventricle and sometimes also in the superior vena cava. These appear as sections of the lead which are wider and more radiodense

4. Estimate the likelihood of EMI based on the characteristics of the proposed surgery and the specific CRD. It is essential to know the location of the CRD and the lead configuration to be able to assess this.

- EMI is more likely if surgery $<15 \mathrm{~cm}$ from CRD or leads (i.e., intrathoracic, shoulder, etc) ${ }^{11}$ or above the umbilicus;

- EMI is more likely if monopolar cautery rather than bipolar cautery to be used;

- EMI is more likely if long ( $>$ five seconds) or frequent ( $<$ five seconds between) bursts of cautery to be used; and

- EMI is more likely if the CRD has unipolar leads or bipolar leads programmed in unipolar mode or with very high sensitivity.

5. Determine the potential for CRD-related complications based on patient- and device-specific factors.

- Patients who are highly pacemaker-dependent are at risk of intraoperative asystole if EMI results in inappropriate inhibition of pacing;

- Patients with ICDs are at risk of inappropriate shocks or ATP if EMI results in inappropriate sensing; and

- Patients who are highly pacemaker-dependent with a unipolar pacemaker on the same side as a surgery known to induce pneumothorax (i.e., pneumonectomy, lobectomy, etc) are at risk of asystole due to a sudden increase in pacing impedance. High 


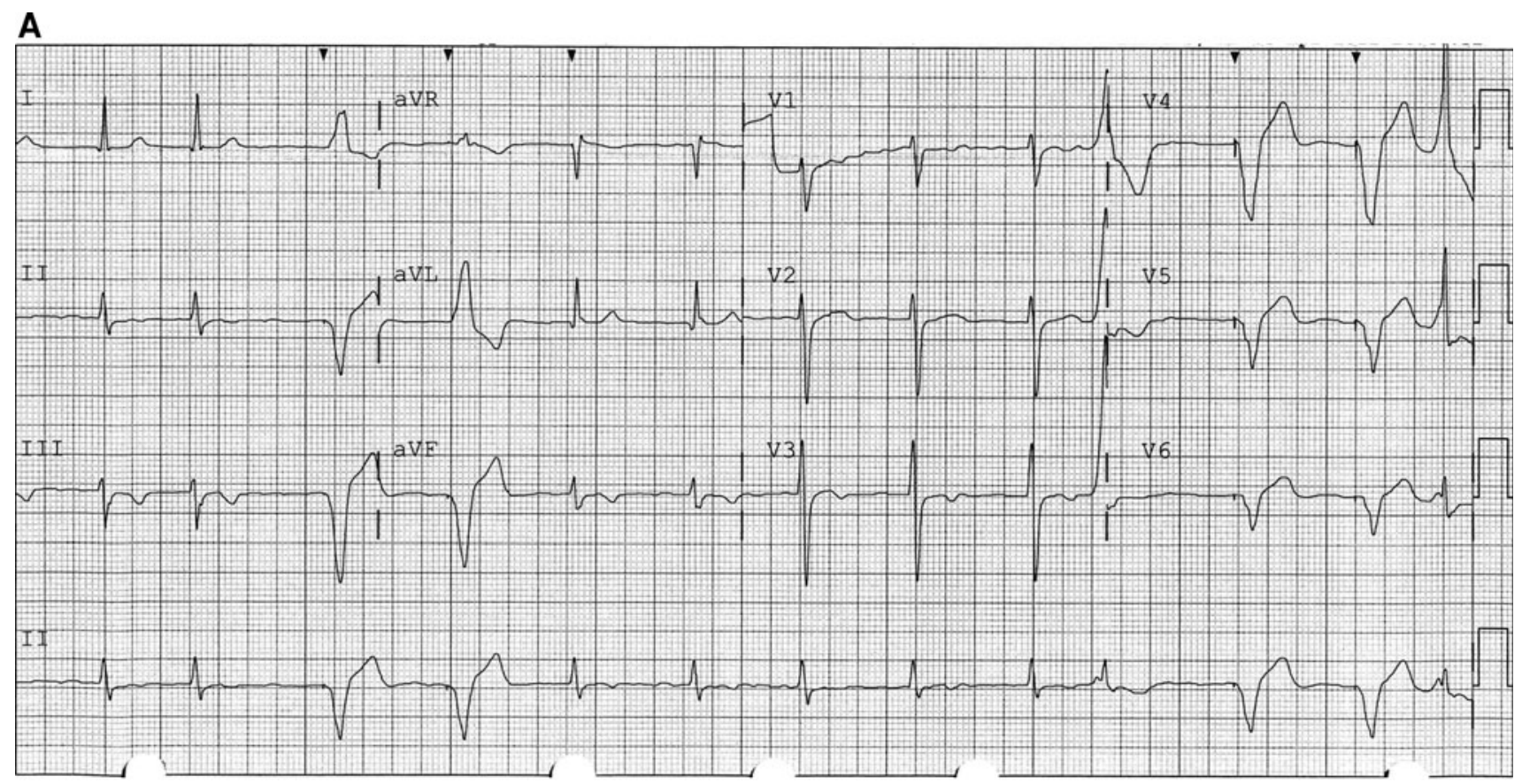

B

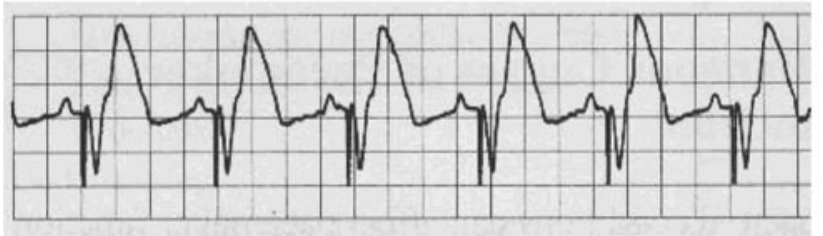

Fig. 2 (A) Electrocardiogram (ECG) showing intermittent right ventricular pacing and underlying atrial fibrillation; and (B) showing continuous p-wave synchronous ventricular pacing

defibrillation thresholds have also been reported in ICD recipients in those circumstances.

6. Develop a plan to minimize the risk of adverse CRDrelated outcomes in conjunction with the surgeon, anesthesiologist, and CRD clinic and/or physician (see "Recommendations for device management" section).

7. If a patient is being considered for elective surgery and has a CRD which has reached the point where replacement is recommended, if at all possible, the CRD should be replaced before elective surgery.

8. Whenever possible, deliver quality healthcare in the patient's community of residence. It is this committee's view that applying the principles of this document carefully should allow for safe management of the majority of patients in their local community. On occasion, it may be appropriate to refer the patient to another institution for surgery to facilitate appropriate perioperative management of their CRD.

\section{Rate modulation technology (rate response)}

Rate modulation technologies were developed in the 1970 s to mimic physiological heart rate increases in response to exercise. While controversy remains relative to the clinical usefulness of universally applied rate-modulated pacing, virtually all pacemakers implanted today have rate modulation functions available. ${ }^{12,18} \mathrm{~A}$ variety of technologies have been investigated and 4 main technologies remain (Table 3). ${ }^{19}$

Various intraoperative events may cause interference with the intended interpretation of physiological changes for rate modulation, ${ }^{20,21}$ and pacemaker-driven tachycardia is described from different sources. Minute ventilation sensors, which may erroneously interpret the signals generated by certain physiological monitors, such as the Agilent/Philips ${ }^{22}$ devices, have been reported to cause tachycardia. Similarly, myoclonia has been reported to be associated with pacemaker-driven tachycardia, presumably by misinterpretation of muscle activity. ${ }^{23}$ 
Table 3 Rate response technologies

\begin{tabular}{ll}
\hline Sensor & Technology \\
\hline Activity sensor & Measures mechanical stress to piezoelectric crystal as a result of motion or acceleration \\
Minute ventilation sensor & Measures transthoracic impedance change between pacemaker lead and pulse generator \\
QT-interval-based sensors & Measures evoked QT interval changes as estimate of adrenergic tone \\
Contractility sensors, activity sensor-based & Measures peak endocardial acceleration as estimate of contractility and global LV function
\end{tabular}

$\mathrm{LV}=$ left ventricular. Adapted from Kaszala and Ellenbogen ${ }^{19}$

Interference with anesthesia management from rate modulation technologies is uncommon but can be clinically confusing, and adverse clinical outcomes may be seen on rare occasions. In the case of elective surgery, it is reasonable to consider suspending rate modulation functions. ${ }^{8}$

\section{Intraoperative care}

In addition to comprehensive general intraoperative care, the management of patients with a CRD requires constant awareness of the unique issues related to their CRD and the availability of specific equipment and trained personnel (Table 4). The anesthesiologist should review and document the perioperative management plan for the device. The patient should have continuous electrocardiographic and pulse monitoring, and the facility should be equipped and the patient prepared for immediate institution of transcutaneous pacing, external defibrillation, and magnet application, should the need arise (Fig. 3).

To minimize EMI with CRDs, the following general principles apply:

1. Bipolar cautery should be used, if possible (appreciating that its use is limited), or the current path of unipolar cautery should be directed away from the CRD and leads;

2. Cautery should be used in short ( $<5$ second) bursts, if possible, allowing for $>5$ seconds between bursts;

3. The lowest acceptable cautery settings should be employed;

4. Cautery must not come into direct contact with CRD hardware; and

5. When the argon beam coagulation system is used, reprogramming the CRD in the dependent patient

Table 4 Intraoperative care

- Review management plan

- Electrocardiographic monitoring

- Prepare resuscitation equipment

- Minimize electromagnetic interference

- Reprogram device, or apply magnet appropriately

- Arrange postoperative management and reprogramming

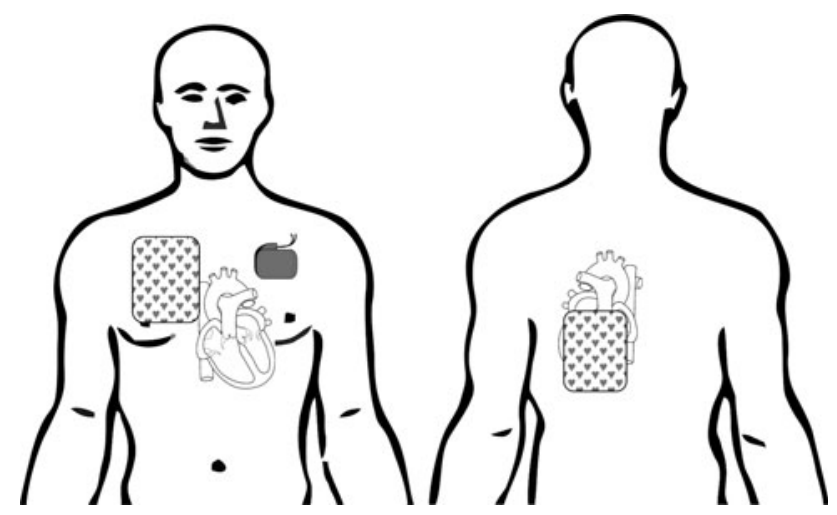

Fig. 3 Placement of transcutaneous pads to facilitate intraoperative external pacing, cardioversion, or defibrillation. Care should be taken to ensure pads are not within 4 to $5 \mathrm{~cm}$ of the implanted pacemaker or defibrillator

should be considered. The argon coagulation system is one in which electrosurgical coagulation is produced by a jet of ionized argon gas encompassing an electrofulguration arc, and it is thought to have the same EMI implications as conventional electrocautery. Interference with a pacemaker has been reported in a case where argon coagulation was used during hepatectomy ${ }^{24}$ and as the use of the argon coagulation system is not amenable to "short bursts", reprogramming should be considered in the pacemakerdependent patient.

Wherever reasonable, this position statement advocates the use of magnet application to disable ICD therapies during surgery (when magnet function has not been disabled during device programming) rather than reprogramming. An abundance of clinical anecdotes of fatal outcomes and close calls associated with the failure to restore appropriate device settings prior to discharge speak to the importance of this issue. ${ }^{16}$ Though this risk is understated in the medical literature, failure to comply places the patient at risk of death if the ICD therapies are not reprogrammed "ON" after surgery. In the event of dysrhythmias requiring therapy, the exact prior outpatient settings are restored immediately upon magnet removal (with specific exceptions, see "Principles of magnet use" section), and the device will function as programmed. 
Magnet placement may not be adequate in specific circumstances (ie, prone or lateral positioning or when the device is incidentally within the surgical field). In these cases, temporary reprogramming to disable ICD therapies should be considered and alternative plans for potentially required defibrillation therapy must be undertaken. Furthermore, there must be a clear plan for postoperative restoration of device programming which can accommodate last-minute changes, such as a delay or cancellation of surgery or same-day discharge. The physician ordering the reprogramming, NOT the industry representative, has the responsibility to ensure that the device is returned to its original settings.

\section{Postoperative care}

The postoperative management of a patient's CRD should continue until the patient has recovered hemodynamically from surgery and until the respective device has been restored to appropriate outpatient settings (Table 5). If a decision is made to disable ICD therapies for ventricular tachycardia (VT) and/or ventricular fibrillation (VF) for surgery or to change the pacing mode to avoid the effects of EMI, it is of CRITICAL importance that a physician is responsible for ensuring that these therapies are reinstated before ECG monitoring is discontinued. In very few cases, the device may emit a persistent tone following magnet removal. This occurrence may indicate an issue with the $\mathrm{CRD}$, and it should be checked or interrogated prior to the patient's discharge from the monitored unit.

Particular care should also be taken when nonhospital personnel (i.e., ICD industry representatives) are asked to reprogram devices at hospitals where no hospital personnel are available to perform this task. Physicians caring for CRD patients in these settings should be aware that industry representatives may not be immediately available to reprogram the device and, as noted, the physician ordering the reprogramming, NOT the industry representative, assumes the responsibility to ensure that the device is returned to its original settings. A clear communication between the industry representative and the ordering

Table 5 Postoperative care

- Monitor ECG until hemodynamically stable

- Reprogram device immediately postoperatively if programming changed

- Analyze lead position and function after thoracotomy

- Recognize that pacemaker-dependent patients may have a different physiologic response to shock

$\mathrm{ECG}=$ electrocardiogram physician should be documented in the chart to ensure proper management of these patients.

Following thoracotomy or cardiac surgery, the position of the leads, especially the atrial lead if present, may change due to cardiac manipulation during surgery. Postoperative device assessment is required to determine appropriate lead function and appropriate output programming. If a change in lead function is determined, this can usually be managed acutely with device programming changes, but it may require lead revision once the patient has recovered from the acute phase of surgery.

For higher-risk surgeries (cardiac, vascular, etc), for which patients are typically managed postoperatively in an intensive care setting, other issues related to CRD management may arise.

1. In patients whose device employs a minute ventilation sensor, inappropriate rapid pacing may result if mechanical ventilator settings cause hyperventilation. Such sensor-related pacing functions can be disabled by reprogramming if such tachycardia is undesired.

2. Patients who are highly pacemaker-dependent will not mount a tachycardia response to hypotension which results from hypovolemia or sepsis. In such patients who are paced $100 \%$ of the time, it may be desirable to reprogram the lower pacing rate to a higher rate more appropriate to the underlying hemodynamic status. If an increased heart rate is required emergently and reprogramming is not immediately available, then placing a magnet may help. This step will typically accelerate pacing to the magnet rate, which is devicespecific but often $>85$ beats $\mathrm{min}^{-1}$. For devices with accelerometer sensors, repetitive tapping over the CRD generator will usually cause acceleration of the paced heart rate to the upper sensor rate (typically 110 to 130 beats $\cdot \mathrm{min}^{-1}$ ).

3. Care should be taken in dealing with cardiogenic shock. In this setting, although acceleration of heart rate may appear to increase cardiac output, it does so at the expense of an increased demand for myocardial oxygen. Several clinical trials have shown that increasing the frequency of right ventricular pacing rate fails to improve outcomes and, in fact, increases the risk of heart failure and death. ${ }^{25,26}$ Although increasing the ventricular pacing rate may be appropriate for specific individuals with noncardiogenic shock who are already $100 \%$ ventricular paced or who have cardiac resynchronization devices, it is almost never appropriate for patients with intrinsic conduction who have standard pacemakers or defibrillators. If the patient is paced only in the atrium, then increasing the atrial pacing rate while minimizing ventricular pacing could be appropriate. 
4. Finally, patients managed postoperatively in an intensive care setting will have continuous cardiac rhythm monitoring. This will allow some flexibility in the timing to restore outpatient settings on CRDs which have been reprogrammed. Although this flexibility is desirable with respect to the availability of CRD clinic personnel, there should be an established protocol in place to ensure that all CRDs are programmed appropriately prior to discharging the patient from a monitored setting. As well, communication should flow back to the patient's usual CRD clinic and/or physicians regarding any permanent changes that have been made to the original programming of their devices.

\section{Principles of magnet use}

\section{Pacemakers}

The response to magnet placement (rate, mode, and output) is manufacturer- and model-specific, and some devices can be programmed to have no response to magnet placement (Table 6). The function of magnet placement is both diagnostic and therapeutic; hence, the battery capacity of most devices can be inferred from the response to magnet placement. Most modern pacemakers will respond to a magnet by pacing asynchronously as long as the magnet is applied; however, in specific instances, notably Intermedics (now Boston Scientific) and Biotronik devices, where the pacemaker responds in a different fashion, a device specialist could be required to interrogate the device.

There are some commonly held misconceptions about magnet placement though adverse events are considered rare in clinical practice:

1. The theoretical risk of sustained R-on-T arrhythmia when a device is programmed to the asynchronous mode (magnet or programming) is considered to be vanishingly low. ${ }^{27}$
2. Rare instances of unintended device reprogramming have been reported associated with the confluence of end-of-life battery condition, magnet movement, and simultaneous electrocautery interference. The device should be interrogated if there is concern that such a situation has been encountered.

3. The effect of magnet application is terminated immediately upon removal of the magnet. There are rare reports of device failure with magnet application.

Of importance, application of a magnet will ensure pacing in pacemaker-dependent patients if EMI inhibits pacing during electrocautery use in surgery. Magnet application could also be useful in other circumstances (eg, when oversensing inhibits pacing or when terminating a pacemaker-mediated tachycardia).

\section{ICDs}

Current ICD models have two distinct functions (i.e., tachyarrhythmia detection and treatment and conventional bradyarrhythmia pacing therapy). The application of a magnet over an ICD will suspend tachyarrhythmia detection and treatment while the magnet is positioned over the device (Table 7), except in devices where this function is specifically programmed off. ${ }^{28}$ Conversely, the magnet will not affect the pacing capability of the ICD. Consequently, appropriate positioning of a magnet over an ICD will cause the device to ignore EMI (and true tachyarrhythmias) and no ICD therapies will be delivered; however, EMI may inhibit the function of the pacemaker component of the device and cause asystole.

Upon magnet application, some devices may emit a tone for a few seconds, or they may emit a tone with each QRS complex sensed by the device. If the device continues to emit a tone once magnet application is removed, there may be an issue with the device (on rare occasions, devices have had "reed switch" faults) and it should be interrogated or checked prior to the patient leaving a monitored environment.

Table 6 Magnet response: pacemakers

\begin{tabular}{|c|c|}
\hline \multicolumn{2}{|l|}{ Pacemaker persists in asynchronous mode } \\
\hline Boston Scientific (Guidant) & Asynchronous at 100 beats $\cdot \mathrm{min}^{-1}, 90$ beats. $\min ^{-1}$, or 85 beats. $\min ^{-1}$ \\
\hline Medtronic & Two beats at 100 beats $\cdot \min ^{-1}, 1$ beat at 90 beats $\cdot \min ^{-1}$, then 85 beats $\cdot \min ^{-1}$ \\
\hline Sorin/ELA & Asynchronous at $98-82$ beats $\cdot \min ^{-1}$ (depending on battery life) \\
\hline St Jude Medical & $\begin{array}{l}\text { Three beats at } 100 \text { beats } \cdot \min ^{-1} \text { or } 98 \text { beats } \cdot \min ^{-1} \text {, then } 85 \text { beats } \cdot \min ^{-1} \text { until magnet } \\
\text { removed }\end{array}$ \\
\hline \multicolumn{2}{|l|}{ Pacemaker reverts to programmed mode } \\
\hline Biotronik & $\begin{array}{l}\text { Ten beats asynchronous at } 90 \text { beats. } \mathrm{min}^{-1} \text { or } 80 \text { beats. } \min ^{-1} \text {, then subsequent at } \\
\text { programmed rate less } 11 \%\end{array}$ \\
\hline $\begin{array}{l}\text { Intermedics (most models; now owned by Boston } \\
\text { Scientific) }\end{array}$ & Transient magnet rate (sometimes 64 beats $\cdot \mathrm{min}^{-1}$ ) then reverts to programmed rate \\
\hline
\end{tabular}

Responses may be different if battery generator replacement is indicated (ERI) or battery depleted (EOL). EOL $=$ end-of-life; ERI $=$ elective replacement indicator 
Table 7 Magnet response: ICDs
Biotronik When a magnet is applied, tachyarrhythmia therapy and detection will be suspended and rate response is suspended.
Bradycardia pacing is not affected by a magnet placed over the device and must be reprogrammed if asynchronous pacing is needed.
Boston If "ENABLE MAGNET USE" is "on"(nominal), device will emit beeping synchronous tones on the R wave. inhibit therapy.
Scientific - If the beeping does not change to a continuous tone after $30 \mathrm{sec}$, the magnet must be taped over the device to temporarily
- If beeping changes to a continuous tone after $30 \mathrm{sec}$, tachy mode has gone to "off" and magnet can be removed.
To turn device back to Monitor and Therapy, magnet should be placed back over the device for 30 seconds until $\mathrm{R}$ wave synchronous tones are heard.
- Magnet application does not affect pacing mode and/or rate.
- If "ENABLE MAGNET USE" is programmed "off" (nominally "on"), then a magnet will NOT inhibit therapy. No tones will be emitted, and a programmer will be needed to turn device off.
Medtronic VF, VT, and FVT detection is suspended. Patient Alert audible tones will occur if applicable and enabled.
Bradycardia pacing is not affected by a magnet placed over the device and must be reprogrammed if asynchronous pacing is needed.
Sorin/ELA When magnet is applied, it disables tachyarrhythmia therapy and arrhythmia detection. Bradycardia function is to pace in the programmed mode at the magnet rate (corresponding to battery voltage); pacing outputs are set to maximum; rate hysteresis and AV extension are set to zero; AV delay is set to the programmed AV delay at rest.
St Jude
Medical
Two programmable options for magnet response: NORMAL (nominal) or IGNORE.
- In "NORMAL" response, magnet blinds detection and delivery of therapy when it is placed over the ICD. Bradycardia pacing is not affected by a magnet placed over the device and must be reprogrammed if asynchronous pacing is needed.
- If "IGNORE" is programmed, magnet application does not disable tachycardia therapies and does not affect pacing mode and/ or rate.

$\mathrm{AV}=$ atrioventricular; ICDs = implantable cardioverter-defibrillators; FVT $=$ fast ventricular tachycardia; VF $=$ ventricular fibrillation; $\mathrm{VT}=$ ventricular tachycardia

Reproduced from Hayes and Friedman ${ }^{28}$ with permission from John Wiley and Sons. ( 2000, 2008 by Mayo Foundation for Medical Eduction and Research

In some devices, unexpected magnet function may occur. Communication with the patient's usual physician and/or clinic can help determine how the device is expected to function with magnet application. Some devices may have their magnet response turned off, in which case, the magnet will not inhibit therapies. In emergent situations where the device response to the magnet cannot be determined, careful monitoring is required during the procedure to determine the response of the device to the EMI (cautery). If inappropriate therapies, such as shock or rapid ATP, should occur, shorter bursts of cautery with $>$ five-second pauses between bursts or switching to bipolar cautery may be required.

If a patient develops VT or VF while the ICD has been disabled by a magnet, the arrhythmia can be managed by removal of the magnet (which will allow the ICD to deliver therapy) or by delivery of an external defibrillator shock. If the use of external pacing or defibrillation is considered necessary, pads should be placed on the patient in the posteroapical fashion, ensuring the apical pad is at least $5 \mathrm{~cm}$ from the location of the CRD (Fig. 3).

\section{Near-future advances: remote device monitoring}

One of the main challenges in the perioperative management of patients with CRDs is having knowledgeable staff and necessary equipment for CRD management situated in a physically remote location from the site where perioperative care is delivered and/or not having personnel readily available when their assistance is needed most. Advances in device management simplify this process, and in this section, we describe the newer modality of remote device monitoring.

The conventional method used by follow-up staff to interact with an implanted device is through use of a device programmer-a laptop computer-sized device which has a radiofrequency wand or "header" extension that is held over the implanted CRD as the programmer uploads information from the device and downloads programming commands to it. In more recent CRD models, the need for a header has been eliminated, and communication between the programmer and the CRD is via short-range wireless telemetry, although the programmer and staff must remain in the same room. The programmer allows interrogation of the CRD to obtain device status information, and it can also send commands to program CRD settings. 
In the past several years, most device manufacturers have developed and introduced telecommunication systems that facilitate CRD status information gathering without the need for a device programmer or for patients to travel to the outpatient device follow-up clinic. These are known collectively as "remote monitoring" systems. While there may be differences amongst the manufacturers, they share common features. The CRD patients are provided with a home base unit (similar to a modem) which is connected to the internet (via a standard phone line connection or even a wireless cell phone communication technology) and communicates wirelessly with the implanted device. Under predefined conditions or at scheduled times, the base unit retrieves information from the CRD which it transmits to designated servers. Authorized personnel use standard web browser software to access these servers and view the information whenever desired. Current remote monitoring systems allow CRD information to be downloaded but do not permit device programming.

Remote monitoring offers opportunities to overcome some of the current challenges of perioperative device management. The following is an example of one possible strategy: a remote monitoring base unit is placed strategically within the operative or perioperative care area; devices capable of remote monitoring are interrogated before and after the surgical procedure, and then the CRD information is immediately available to staff for review. Some manufacturers have implemented remote monitoring in such a manner that each patient receives a base station specific to their device which cannot be used by any other device. In such circumstances, the patient must bring their own station to the surgical centre. Other administrative and technological issues, such as a telephone line connection, would need to be addressed in such a strategy. A communication protocol should also be established to ensure that perioperative and CRD staffs communicate effectively about the patient and the CRD status. If any CRD anomalies are identified, CRD staff would be dispatched to address the issues; otherwise, the patient could be discharged from the perioperative care area without further concern about the CRD. In this way, CRD specialists can provide the best advice to the surgeons and anesthesiologists caring for the patient. This is a prime example illustrating how CRD technological advances can potentially change the existing paradigm of the perioperative management of CRD patients.

\section{Recommendations for device management}

Whenever possible, planning for perioperative device management should be a collaborative process between the patient's CRD clinic and/or physician and the operative team. Guided by the following recommendations, this approach will ensure optimum planning to meet the patient's specific needs.

Pacemakers

1. Operations with minimal or no electrocautery

- No change to pacemaker programming; have magnet available.

2. Operations with significant or unavoidable electrocautery

A. Patient is pacemaker dependent

- If device is continuously accessible and visible and device responds continuously to magnet placement, use magnet to initiate asynchronous pacing.

- If device is not accessible or device does not respond continuously to magnet placement, then reprogram device to asynchronous mode at the start of the procedure.

B. Patient is not pacemaker dependent

- If device is continuously accessible and visible and device responds continuously to magnet placement, have magnet available to intervene if necessary.

- If device is not continuously accessible and operative circumstances require a more physiologic rate, then consult with the CRD clinic to consider reprogramming to a physiologically acceptable rate for the duration of the procedure.

3. Consider suspending rate modulation therapies if enabled. 
Bradycardia function of ICD

1. Operations with minimal or no electrocautery

- No change to pacemaker programming.

2. Operations with significant or unavoidable electrocautery

A. Patient is pacemaker dependent

- Consider reprogramming device to asynchronous mode prior to procedure.

B. Patient is not pacemaker dependent

- Consider reprogramming to physiologically acceptable rate in synchronous mode for the duration of the procedure.

Tachyarrhythmia functions of ICD

1. Operations with no electrocautery

- No change to VT and/or VF programming; have magnet available to suspend tachyarrhythmia functions if necessary.

2. Operations with electrocautery

A. Device accessible and clear of operative field

- Position magnet over device during surgical procedure.

B. Device is not accessible or in operative field or magnet cannot be securely affixed in a satisfactory position

- Reprogram to defeat tachyarrhythmia therapies; apply external defibrillator pads and ensure postoperative reprogramming to baseline therapies prior to removal of external defibrillator apparatus and discontinuation of electrocardiographic monitoring.

\section{Considerations for the pediatric patient with an implanted CRD}

Although it is relatively uncommon for pediatric patients to have an implanted CRD compared with the adult population, such patients do present with the need for operative management. While all of the general principles in this document also apply to pediatric patients, there are a several important issues to emphasize in managing a pediatric patient with a CRD perioperatively.

There are relatively fewer centres that manage pediatric CRD and therefore, for elective cases, management at their home centre, or one familiar with their underlying cardiac condition, is likely most appropriate. For emergency surgery where this is not possible, communication with the tertiary centre managing the CRD is imperative. The most common indication for pacing in the pediatric population is complete heart block following cardiac surgery. Because congenital heart disease frequently exists in the presence of other organ involvement, these individuals may be required to undergo noncardiac surgery. These patients usually have competent sinus node function and thus can respond to the stress of the intervention appropriately; however, upper rate behaviour programming is particularly important in young patients, whose sinus rates will approach the upper capacity of the device when the patient is stressed. Such complex patients usually have epicardial devices and leads and familiarity with the location of these is essential before embarking on surgery.

\section{Noncardiac electrical devices}

Noncardiac IEDs are being used increasingly as therapeutic modalities for a wide range of disorders. ${ }^{4-6}$ Examples of commonly used IEDs include deep brain stimulators, spinal cord stimulators, vagal and phrenic nerve stimulators, and gastric stimulators. Many of the issues of perioperative management are similar to those for cardiac devices, though there are specific differences.

The IED consists of two components (i.e., a pulse generator [battery powered] and electrodes implanted in the target neural tissue). In contrast to cardiac pacemakers, patients can turn off some of these devices with an external 
remote. Preoperative considerations include identification and localization of the devices and the status of the severity of the patient's symptoms when the device is turned offdeactivation of the device in a Parkinson's patient may result in severe symptoms.

Interactions between common medical equipment may occur. Intraoperative electrocautery has the potential to burn neural tissue around the stimulator or to reprogram the IED. Turning off the device may decrease damage to the stimulator, and therefore this is recommended for operative procedures where cautery is required. In many situations, patients themselves can turn off their device, using a patientspecific controlling device. Use of bipolar electrocautery is safer, but if monopolar cautery is required, the return electrode should be placed as far away as possible from the IED to conduct the current away, and the lowest possible source of energy should be used in short irregular pulses. Short wave diathermy modalities should not be used as they produce radio frequency currents and heating of electrodes.

The safe use of external and internal cardiac defibrillators has not been established in the presence of IEDs. Defibrillation may impair IED function, and cardioversion may cause lesions around the target area. If cardioversion or defibrillation is required, the paddles must be positioned as far away as possible from the IED and placed perpendicular to the IED using the lowest clinically appropriate energy output. The function of the IED needs to be checked after such treatment. As both cardiac and noncardiac generators can be affected by the placement of a magnet over them, it may be useful to stimulate each unit separately to identify the presence of significant interference. Some IEDs may produce artifacts and interfere with the recording of an ECG. Electroconvulsive therapy, radiofrequency neuroablation, and peripheral nerve stimulation have been reported to be safe by switching off the stimulator and placing the probes away from the IED.

\section{Industry resources}

Consolidation in the industry has resulted in the production of the majority of pacemakers in North America by five major companies. For further information or locations of technicians for pacemaker devices, each company offers a 24-hr help line; telephone numbers are listed below.

- Biotronik +1-800-547-0394 (http://www.biotronik.com/ en/ca/home\#)

- Boston Scientific (Guidant, Telectronics) +1-800-CAR DIAC (+1-800-227-3422) (http://www.bostonscientific. com)

- Medtronic +1-800-MEDTRONIC (+1-800-633-8766) (http://www.medtronic.com)
- St Jude Medical +1-800-722-3774 http://www.sjm professional.com)

- Sorin/ELA +1-800-352-6466 (USA, 24-hour) (http:// www.sorin-crm.com)

St Jude Medical maintains an on-line reference of CRDs from all companies: (http://www.sjmprofessional.com/ $\mathrm{drg} /$ device-reference-guide.aspx), as does Boston Scientific (http://www.bostonscientific.com/templatedata/imports/ HTML/PPR/ppr/index.shtm

\section{References}

1. Birnie D, Williams $K$, Guo A, et al. Reasons for escalating pacemaker implants. Am J Cardiol 2006; 98: 93-7.

2. Rozner MA. The patient with a cardiac pacemaker or implanted defibrillator and management during anesthesia. Curr Opin Anaesthesiol 2007; 20: 261-8.

3. Curtis JP, Leubbert JJ, Wang Y, et al. Association of physician certification and outcomes among patients receiving an implantable cardioverter-defibrillator. JAMA 2009; 301: 1661-70.

4. Farris S, Giroux M. Deep brain stimulation: a review of the procedure and the complications. JAAPA 2011; 24(39-40): 42-5.

5. Poon CC, Irwin MG. Anaesthesia for deep brain stimulation and in patients with implanted neurostimulator devices. $\mathrm{Br} \mathrm{J}$ Anaesth 2009; 103: 152-65.

6. Venkatraghavan L, Chinnapa V, Peng P, Brull R. Non-cardiac implantable electrical devices: brief review and implications for anesthesiologists. Can J Anesth 2009; 56: 320-6.

7. American Society of Anesthesiologists Task Force on Perioperative Management of Patients with Cardiac Rhythm Management Devices. Practice advisory for the perioperative management of patients with cardiac rhythm management devices: pacemakers and implantable cardioverter-defibrillators: a report by the American Society of Anesthesiologists Task Force on Perioperative Management of Patients with Cardiac Rhythm Management Devices. Anesthesiology 2005; 103: 186-98

8. American Society of Anesthesiologists. Practice advisory for the perioperative management of patients with cardiac implantable electronic devices: pacemakers and implantable cardioverterdefibrillators: an updated report by the American Society of Anesthesiologists Task Force on Perioperative Management of Patients With Cardiac Implantable Electronic Devices. Anesthesiology 2011; 114: 247-61

9. Crossley GH, Poole JE, Rozner MA, et al. The Heart Rhythm Society (HRS)/American Society of Anesthesiologists (ASA) Expert Consensus Statement on the perioperative management of patients with implantable defibrillators, pacemakers and arrhythmia monitors: facilities and patient management. Heart Rhythm 2011; 8: 1114-54.

10. Medicines and Healthcare Products Regulatory Agency. Guidelines for implantable cardioverter defibrillators (ICDs) - pacemaker perioperative management. Available from: http://www.mhra.gov.uk/ Safetyinformation/Generalsafetyinformationandadvice/Productspecificinformationandadvice/Product-specificinformationand advice-A-F/Cardiacpacemakersanddefibrillators\%28implantable $\% 29 /$ Guidelinesforimplantablecardioverterdefibrillators-pace makerperioperativemanagement/CON2023432 (accessed April 2011)

11. Cheng A, Nazarian S, Spragg DD, et al. Effects of surgical and endoscopic electrocautery on modern-day permanent pacemaker and implantable cardioverter-defibrillator systems. Pacing Clin Electrophysiol 2008; 31: 344-50. 
12. Stone ME, Apinis A. Current perioperative management of the patient with a cardiac rhythm management devices. Semin Cardiothorac Vasc Anesth 2009; 13: 31-43.

13. Pinski SL, Trohman RG. Interference in implanted cardiac devices, part II. Pacing Clin Electrophysiol 2002; 25: 1496-509.

14. Sponga $S$, Mascioli $G$, Voisine P, Vitali E. A case of inefficient defibrillation during thoracotomy. J Card Surg 2011; 26: 338-9.

15. Cohen TJ, Lowenkron DD. The effects of pneumothorax on defibrillation thresholds during pectoral implantation of an active can implantable cardioverter defibrillator. Pacing Clin Electrophysiol 1998; 21: 468-70.

16. Boston Scientific. Latitude Patient Management System. Boston: Boston Scientific, 2008. Available from URL: http://www.boston scientific.com/templatedata/imports/Microsite/cardiac-rhythm-resources/ patient_education_downloads/c7-135_welcome_checklist.pdf (accessed December 2011)

17. Arnold RW, Jensen PA, Kovtoun TA, Maurer SA, Schultz JA. The profound augmentation of the oculocardiac reflex by fast acting opioids. Binocul Vis Strabismus Q 2004; 19: 215-22.

18. Lamas GA, Knight JD, Sweeney $M O$, et al. Impact of rate-modulated pacing on quality of life and exercise capacity-evidence from the Advanced Elements of Pacing Randomized Controlled Trial (ADEPT). Heart Rhythm 2007; 4: 1125-32.

19. Kaszala K, Ellenbogen KA. Device sensing: sensors and algorithms for pacemakers and implantable cardioverter defibrillators. Circulation 2010; 122: 1328-40.

20. Anand NK, Maguire DP. Anesthetic implications for patients with rate-responsive pacemakers. Semin Cardiothorac Vasc Anesth 2005; 9: 251-9.

21. Wong DT, Middleton $W$. Electrocautery-induced tachycardia in a rate-responsive pacemaker. Anesthesiology 2001; 94: 710-1.

22. Hu R, Cowie DA. Pacemaker-driven tachycardia induced by electrocardiograph monitoring in the recovery room. Anaesth Intensive Care 2006; 34: 266-8.
23. Altose MD, Leon-Ruiz, E. Etomidate-induced pacemaker-mediated ventricular tachycardia. Anesthesiology 2007; 106: 1059-60.

24. Werner P, Charbit B, Samain E, Farah E, Marty J. Interference between a dual-chamber pacemaker and argon electrocautery devices during hepatectomy (French). Ann Fr Anesth Reanim 2001; 20: 716-9.

25. Wilkoff BL, Cook JR, Epstein AE, et al. The DAVID Trial Investigators. Dual-chamber pacing or ventricular backup pacing in patients with an implantable defibrillator: the Dual Chamber and VVI Implantable Defibrillator (DAVID) Trial. JAMA 2002; 288: 3115-23.

26. Sweeney MO, Hellkamp AS, Lee KL. Mode Selection Trial (MOST) Investigators. Association of prolonged QRS duration with death in a clinical trial of pacemaker therapy for sinus node dysfunction. Circulation 2005; 111: 2418-23.

27. Filipovic M, Michaux I, Seeberger MD. Harm associated with reprogramming pacemakers for surgery. Anesthesiology 2002; 97: 1033-4.

28. Hayes DL, Friedman PA. Electromagnetic interference and implantable devices. In: Hayes DL, Friedman PA, editors. Cardiac Pacing, Defibrillation and Resynchronization: A Clinical Approach. 2nd ed. Hoboken: Wiley-Blackwell; 2008. p. 550-72.

\section{Disclosures}

Jeff Healey has received research grants (major) from Boston Scientific and St Jude Medical. Stanley Tung has received research grants and unrestricted continuing medical education grants (minor) from Medtronic. Raymond Yee has received research grants (major) from Medtronic. Marianne Beardsall has received research grants (minor) from Medtronic and St Jude Medical. None of the other authors has any conflicts of interest to disclose. 